\title{
Communication
}

\section{Life-times of long-lived coherences under different motional regimes}

\author{
Aurélien Bornet ${ }^{\mathrm{a}}$, Riddhiman Sarkar ${ }^{\mathrm{a}, *}$, Geoffrey Bodenhausen ${ }^{\mathrm{a}, \mathrm{b}}$ \\ ${ }^{a}$ Institut des Sciences et Ingénierie Chimiques, Ecole Polytechnique Fédérale de Lausanne, EPFL, Batochime, 1015 Lausanne, Switzerland \\ b Département de Chimie, associé au CNRS, Ecole Normale Supérieure, 24 Rue Lhomond, 75231, Paris Cedex 05, France
}

\section{A R T I C L E I N F O}

\section{Article history:}

Received 23 April 2010

Revised 24 May 2010

Available online 8 June 2010

\section{Keywords:}

Coherent superpositions

Transverse relaxation

Spectral density

\begin{abstract}
A B S T R A C T
The transverse relaxation rate $R_{2}$ of single quantum coherences, the relaxation rate $R_{L L C}$ of long-lived coherences (LLC), and the ratio $R_{2} / R_{L L C}$ have been studied by experiment, simulation and theory in the two-spin system formed by the Glycine aliphatic protons of the dipeptide Alanine-Glycine as a function of the correlation time of rotational diffusion.
\end{abstract}

(c) 2010 Elsevier Inc. All rights reserved.

\section{Introduction}

Singlet and triplet states [1-3] associated with a pair of magnetically equivalent spins $1 / 2$ belong to different irreducible representations of the permutation group. Transitions between singlet and triplet states are therefore symmetry-forbidden and have vanishing transition probabilities. However, in certain cases, it is possible to excite coherences that span such forbidden transitions. The evolution of such coherences can be monitored indirectly as a function of time by two-dimensional (2D) spectroscopy [4]. It has been shown recently [5] that a coherent superposition of a singlet state $\left|S_{0}\right\rangle$ and a central triplet state $\mid T_{0}>$, known as a long-lived coherence (LLC), can have a very long life-time. Its decay rate $R_{L L C}$ can be significantly slower than the transverse relaxation rate $R_{2}$ of single quantum coherences (SQC) in the same system. This opens new perspectives for homogeneous line narrowing in NMR spectroscopy.

A pair of equivalent spins $1 / 2$ is best described in the singlet-triplet basis (STB) $\Phi_{\mathrm{STB}}=\left\{\left|T_{+1}\right\rangle,\left|T_{0}\right\rangle,\left|S_{0}\right\rangle,\left|T_{-1}\right\rangle\right\}$, where $T_{+1}=|\alpha \alpha\rangle$; $T_{0}=N(|\alpha \beta\rangle+|\beta \alpha\rangle) ; S_{0}=N(|\alpha \beta\rangle-|\beta \alpha\rangle)$; and $T_{-1}=|\beta \beta\rangle$ with $N=2^{-1 / 2}$. A long-lived coherence $Q_{L L C}$ can be defined as a coherent superposition of the singlet state $\mid S_{0}>$ and the central triplet state $\mid T_{0}>$ :

$Q_{L L C}=\left|S_{0}\right\rangle\left\langle T_{0}|+| T_{0}\right\rangle\left\langle S_{0}\right|$

In terms of Cartesian angular momentum operators, $Q_{L L C}$ can be written as:

$Q_{L L C}=\left(I_{x}-S_{x}\right)$

\footnotetext{
* Corresponding author.

E-mail address: riddhiman.sarkar@gmail.com (R. Sarkar).
}

In order to observe a $Q_{L L C}$ in a two-spin system with two magnetically inequivalent nuclei $I$ and $S$, one must first excite a difference $\left(I_{x}-S_{x}\right)$ and then render $I$ and $S$ magnetically equivalent by applying a strong radio-frequency field, in the simplest case a continuous-wave (CW) radio-frequency ( $r f$ ) field with a carrier positioned half-way between the two chemical shifts. Starting from Boltzmann equilibrium $\left(I_{z}+S_{z}\right)$, a semi-selective $(\pi)_{x}$ inversion pulse is first applied to the doublet of spin $S$ to generate $\left(I_{z}-S_{z}\right)$. A non-selective pulse $(\pi / 2)_{y}$ converts this state into $\left(I_{x}-S_{x}\right)$. When a CW field is applied along the $x$-axis of the rotating frame, the chemical shifts of the two spins are in effect suppressed. At this point, the system is best described in $\Phi_{\text {STB }}$ rather than in the product basis. The long-lived coherence $Q_{L L C}$ oscillates with a frequency that corresponds to the separation between the singlet state $\mid \mathrm{S}_{0}>$ and the central triplet state $\mid \mathrm{T}_{0}>$ [5]. If the $r f$ field is sufficiently strong, this splitting is equal to the scalar coupling constant $J_{I S}$. The transverse relaxation occurs with a rate constant $R_{L L C}$, so that the evolution of the long-lived coherence is described by:

$\frac{d}{d t} Q_{L L C}=-\left(R_{L L C}+i \pi J_{I S}\right) Q_{L L C}$

In a two-spin system, the SQC relaxation rate is given by the usual expression:

$R_{2}=\left(\frac{b_{I S}^{2}}{20}\right)[9 J(0)+15 J(\omega)+6 J(2 \omega)]+R_{2}^{e x t}$

where $b_{I S}=-\frac{\mu_{0}}{4 \pi} \frac{\gamma^{2} h}{r^{3}}$ is the strength of the homonuclear dipolar coupling, $\mathrm{r}_{\mathrm{IS}}$ the inter-nuclear distance, $J(\omega)=\frac{\tau_{c}}{1+\omega^{2} \tau_{c}^{2}}$ the spectral density function, and $\tau_{c}$ the rotational correlation time. The term $R_{2}^{\text {ext }}$ comprises contributions from external random fluctuating fields 
(including those arising from dissolved oxygen and other paramagnetic species), the modulations of the chemical shift anisotropies (CSA's), and dipolar interactions with further nuclei $R, R$, etc.

The relaxation rates $R_{L L C}$ of LLC's can be significantly smaller than the transverse relaxation rates $R_{2}$ in the same system. If there are only two spins, the main mechanism that drives relaxation of $R_{L L C}$ arises from dipolar interactions between the two 'active' spins $I$ and $S$. (Note that this stands in contrast to the relaxation rate $R_{L L S}$ of the population of the long-lived state $\left|S_{0}\right\rangle$, for which the dipolar interaction between $I$ and $S$ is completely ineffective.) The relaxation rate constant $R_{L L C}$ can be calculated by Redfield theory [6]. If the $r f$ field is sufficiently strong, it need not be taken into account explicitly and it is sufficient to retain the truncated Hamiltonian where the two spins $I$ and $S$ have the same Larmor frequency. The transverse relaxation rates $R_{L L C}$ of long-lived coherences under strong $r f$ irradiation can be evaluated in analogy to the longitudinal relaxation rates $R_{L L S}$ of long-lived states [7-9]:

$R_{L L C}=\left(\frac{b_{I S}^{2}}{20}\right)[J(0)+3 J(\omega)+6 J(2 \omega)]+R_{L L C}^{e x t}$

Eqs. (4) and (5) were derived assuming isotropic reorientation, and are limited to rigid symmetric molecules, which may not be entirely satisfactory for the applications discussed below. The term $R_{L L C}^{e x t}$ again comprises contributions from external random fluctuating fields arising from dissolved oxygen and other paramagnetic species, the modulations of the chemical shift anisotropies (CSA's) of the two active spins (except if they are completely correlated), and dipolar interactions with further nuclei $R, R$ '. If the external contributions $R_{L L C}^{\text {ext }}$ are small, the long-lived coherence $Q_{L L C}$ has a longer life-time than single quantum coherences. Eqs. (4) and (5) predict that the ratio $R_{2} / R_{L L C}$ varies in the range $3<R_{2} / R_{L L C}<9$ depending on the rotational correlation time (Fig. 1). In the fast tumbling regime $\left(\tau_{c} \ll 1 / \omega_{0}\right)$, the spectral density functions are equal at all frequencies, i.e., $J(0)=J(\omega)=J(2 \omega)$, hence $R_{2} / R_{L L C}=3$. When molecular tumbling slows down, i.e., when $\tau_{c} \gg 1 / \omega_{0}$, the ratio can approach the limit $R_{2} / R_{L L C}=9$.

In this communication, we have sought to verify these predictions by experiments and simulations for the dipeptide Ala-Gly, which is admittedly neither a rigid nor an isotropic rotor. In this system, the two $\mathrm{H}^{\alpha}$ protons of Glycine are diastereotopic with a difference in chemical shifts of $0.1 \mathrm{ppm}(42.7 \mathrm{~Hz}$ at $9.4 \mathrm{~T}$ or $400 \mathrm{MHz}$ for protons) and a scalar coupling $J\left(\mathrm{H}^{\alpha}, \mathrm{H}^{\alpha}\right)=-17.2 \mathrm{~Hz}$ [10]. The experimental decay rates $R_{2}$ and $R_{L L C}$ and the ratio $R_{2} / R_{L L C}$ were compared with numerical simulations as a function of $\tau_{c}$. In the simulations of Fig. 1, the correlation time of rotational diffusion



Fig. 1. Numerical simulations of the ratio $R_{2} / R_{L L C}$ calculated by GAMMA [14] as a function of the rotational correlation time $\tau_{c}$. The two $\mathrm{H}^{\alpha}$ protons of Glycine in the dipeptide Ala-Gly were treated as an isolated two-spin system in a static field $B_{0}=9.4 \mathrm{~T}$ ( $400 \mathrm{MHz}$ for protons), $\Delta v_{I S}=42.7 \mathrm{~Hz}, J_{I S}=17.2 \mathrm{~Hz}, r_{I S}=1.77 \AA$, $r f$ amplitude $v_{1}=1500 \mathrm{~Hz}$, with the $r f$ carrier half-way between the two chemical shifts. Only the dipolar interaction between the two active nuclei $I$ and $S$ was taken into account. was varied in the range $0.01<\tau_{c}<3$ ns. As described by Eqs. (4) and (5), the ratio $R_{2} / R_{L L C}$ is close to 3 for fast motion and approaches 9 asymptotically in the slow motion limit.

Experiments were carried out to measure the ratio $R_{2} / R_{L L C}$ in a sample of $1 \mathrm{M}$ Ala-Gly dissolved in a 3:1 (v/v) $\mathrm{D}_{2} \mathrm{O} /$ DMSO- $_{6}$ mixture, where the viscosity strongly depends on temperature [11]. The relationship between the (microscopic) rotational correlation time $\tau_{c}$ and the (macroscopic) viscosity $\eta$ is given by the StokesEinstein equation [12]. In the numerical simulations, we varied $\tau_{c}$ empirically to match the simulated and experimental $R_{2}$ rates at each temperature. The slow motion regime could not be fully explored because one cannot lower the temperature enough without causing excessive line-broadening, which makes LLC experiments difficult.

The rates $R_{2}$ were measured by on-resonance $R_{1}$ experiments [5] and fitted with $I(t)=I_{0} \exp \left(-R_{2} t_{1}\right)$, assuming that $R_{2}=R_{1}$. The rates $R_{L L C}$ were measured with the pulse sequence described by Sarkar et al. [5] and by fitting the intensities of the signals $\left(I_{x}-S_{x}\right)$ that oscillate as a function of $t_{1}$ with the function $I\left(t_{1}\right)=I_{0} \cos \left(2 \pi J_{I S} t_{1}\right) \exp \left(-R_{L L C} t_{1}\right)$. The role of the external contributions $R_{L L C}^{\text {ext }}$ and $R_{2}^{\text {ext }}$ has been evaluated as follows: The contributions of the chemical shift anisotropies (CSA's) of the two active spins can be neglected for aliphatic $\mathrm{H}^{\alpha}$ protons in Glycine at $400 \mathrm{MHz}$. In the dipeptide Ala-Gly, dipolar interactions to further nuclei $R, R^{\prime}$ may arise from the lone $\mathrm{H}^{\alpha}$ and three $\mathrm{H}^{\beta}$ protons of the neighbouring Alanine residue, the amide proton being replaced by a deuteron in this solvent. It turns out that, because of their distance, these four Alanine protons do not have a significant effect on the rate $R_{2}$ of the two Glycine protons, as evidenced by numerical calculations carried out with and without considering the four Alanine protons. Since the calculation of $R_{L L C}$ rates in a 6-spin system is quite demanding, we have considered a reduced 3-spin system by dropping the three $\mathrm{H}^{\beta}$ protons of the Alanine residue, and found that the ratio $R_{2} / R_{L L C}$ in Glycine is not affected by the remaining Alanine $\mathrm{H}^{\alpha}$ proton, regardless of $\tau_{c}$. We may therefore assume that the ratio $R_{2} / R_{L L C}$ is not affected by the four remote protons in AlaGly.

On the other hand, paramagnetic oxygen was found to yield a significant contribution to $R_{L L C}^{\text {ext }}$. If one does not remove oxygen, the observed experimental ratio was in the range $2.2<R_{2} / R_{L L C}<2.5$ for $0.04<\tau_{c}<0.06 \mathrm{~ns}$ (Fig. 2). After three freeze-pump-thaw cycles under Argon atmosphere, the ratio $R_{2} / R_{L L C}$ increases, particularly in the fast tumbling regime, in comparison to the nondegassed sample (Fig. 2). As the correlation time $\tau_{c}$ increases with

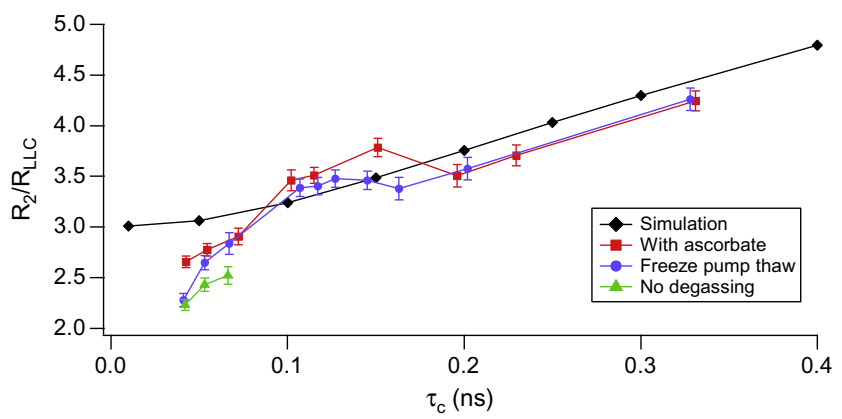

Fig. 2. Simulations of the ratio $R_{2} / R_{L L C}$ calculated by GAMMA [14] as a function of $\tau_{c}$ (black line with diamonds). The same parameters as in Fig. 1 were used. Experimental ratios $R_{2} / R_{L L C}$ in $1 \mathrm{M}$ Ala-Gly dissolved in a $\mathrm{D}_{2} \mathrm{O}$ /DMSO 3:1 mixture without degassing (green triangles), after degassing with three freeze-pump-thaw cycles under Argon (blue circles), and after addition of $20 \mathrm{mM}$ sodium ascorbate as a reducing agent to quench dissolved paramagnetic oxygen (red squares). The experimental points correspond to temperatures between 300 and $248 \mathrm{~K}$. (For interpretation of the references to colour in this figure legend, the reader is referred to the web version of this article.) 
decreasing temperature, the trend of the ratio $R_{2} / R_{L L C}$ agrees qualitatively with the simulations. Paramagnetic oxygen can be quenched by the addition of $20 \mathrm{mM}$ sodium ascorbate (vitamin C) as a reducing agent [13]. Like in the degassed sample, the ratio $R_{2} / R_{L L C}$ follows the simulated curve as $\tau_{c}$ decreases. In the range $0.04<\tau_{c}<0.06 \mathrm{~ns}$, the ratio $R_{2} / R_{L L C}$ is even higher after degassing by freeze-pump-thaw cycles (Fig. 2). Nevertheless, the experimental ratio $R_{2} / R_{L L C}$ remains lower than 9 . This could be due to various contributions to $R_{2}^{e x t}$ and $R_{L L C}^{e x t}$, such as residual paramagnetic oxygen in the sample and other sources of fluctuating fields.

\section{Conclusions}

We have compared the transverse relaxation rates $R_{L L C}$ of longlived coherences with the decay rates $R_{2}$ of single-quantium coherences. It was shown by theory and simulations that the ratio $R_{2} /$ $R_{L L C}$ varies from 3 to 9 as a function of the rotational correlation time $\tau_{c}$. In the extreme narrowing regime, the experimental ratio was found to be about $R_{2} / R_{L L C}=2.6$, somewhat lower than expected, even when paramagnetic oxygen was removed from the sample by freeze-pump-thaw cycles or scavenged by the addition of ascorbate. The slow motion regime could not be fully explored because one cannot lower the temperature below $248 \mathrm{~K}$ without causing excessive line-broadening, but the highest ratio was found to be $R_{2} / R_{L L C}=4.6$. The experiments provide evidence of the efficiency of ascorbate as scavenger of paramagnetic oxygen.

\section{Acknowledgments}

We are indebted to Puneet Ahuja and Paul Vasos for many helpful discussions. Martial Rey provided valuable technical assistance. Pascal Miéville helped with sample preparation. We thank Olivier Wagnières for preliminary measurements. This work was supported by the Swiss National Science Foundation (SNF Grant No. 200020-124694 to G.B. and P. Vasos), the Ecole Polytechnique Fédérale de Lausanne (EPFL), the Swiss Commission for Technology and Innovation (CTI, Grant No. 9991.1 PFIW-IW to G.B., Paul Dyson, Jean-Philippe Ansermet, and Paul Vasos), and the French CNRS.

\section{References}

[1] M. Carravetta, O.G. Johannessen, M.H. Levitt, Phys. Rev. Lett. 92 (2004) 153003.

[2] M. Carravetta, M.H. Levitt, J. Am. Chem. Soc. 126 (2004) 6228.

[3] R. Sarkar, P.R. Vasos, G. Bodenhausen, J. Am. Chem. Soc. 129 (2007) 328.

[4] G. Pileio, M. Carravetta, M.H. Levitt, Phys. Rev. Lett. 103 (2009) 083002

[5] R. Sarkar, P. Ahuja, P.R. Vasos, G. Bodenhausen, Phys. Rev. Lett. 104 (2010) 053001.

[6] A. Redfield, G. IBM J. Res. Dev. 1 (1957) 19.

[7] K. Gopalakrishnan, G. Bodenhausen, J. Mag. Res. 182 (2006) 254

[8] G. Pileio, M.H. Levitt, J. Chem. Phys. 130 (2009) 214501.

[9] R. Sarkar, PhD thesis, Ecole Polytechnique Fédérale de Lausanne, 2009.

[10] R. Sarkar, P. Ahuja, P.R. Vasos, A. Bornet, O. Wagnières, G. Bodenhausen, Progress NMR Spectr., (2010), in press.

[11] S. Cavadini, J. Dittmer, S. Antonijevic, G. Bodenhausen, J. Am. Chem. Soc. 127 (2005) 15744.

[12] S. Cavadini, P.R. Vasos, Concepts Magn. Reson. 32A (2008) 68

[13] P. Mieville, P. Ahuja, R. Sarkar, S. Jannin, P.R. Vasos, S. Gerber, M. Mishkovsky, A. Comment, R. Gruetter, O. Ouari, P. Tordo, G. Bodenhausen, Angewandte Chemie, (2010), in press.

[14] S.A. Smith, T.O. Levante, B.H. Meier, R.R. Ernst, J. Magn. Reson. Ser. A 106 (1994) 75 . 